\title{
The Plasma Levels of the Angiogenic Cytokine Endocan Are Elevated in Patients with Multiple Myeloma
}

\author{
NORMANN STEINER ${ }^{1}$, ROMAN HAJEK ${ }^{2}$, SABINA SEVCIKOVA ${ }^{3,4}$, BOJANA BORJAN ${ }^{1}$, \\ GEROLD UNTERGASSER ${ }^{1,5}$, GEORG GÖBEL $^{6}$ and EBERHARD GUNSILIUS ${ }^{1}$ \\ ${ }^{1}$ Laboratory for Tumor Biology and Angiogenesis, Department of Internal Medicine V \\ (Hematology and Medical Oncology), Innsbruck Medical University, Innsbruck, Austria; \\ ${ }^{2}$ Department of Hemato-oncology, Faculty of Medicine, \\ University of Ostrava and University Hospital Ostrava, Ostrava, Czech Republic; \\ ${ }^{3}$ Babak Myeloma Group, Department of Pathological Physiology, \\ Faculty of Medicine, Masaryk University, Brno, Czech Republic; \\ ${ }^{4}$ Department of Clinical Hematology, University Hospital Brno, Brno, Czech Republic; \\ ${ }^{5}$ Tyrolean Cancer Research Institute, Innsbruck, Austria; \\ ${ }^{6}$ Department of Medical Statistics, Informatics and Health Economics, \\ Innsbruck Medical University, Innsbruck, Austria
}

\begin{abstract}
Background/Aim: Monoclonal gammopathy of unknown significance (MGUS) and smoldering multiple myeloma often precede multiple myeloma (MM). The identification of biomarkers predicting progression to MM might facilitate an earlier diagnosis of MM. Our study assessed the diagnostic value of plasma levels of endocan, a 50-kDa soluble dermatan sulfate proteoglycan produced and secreted by endothelial cells, hitherto unknown in $M M$, in patients with plasma cell dyscrasia. Materials and Methods: Endocan levels were determined in 96 peripheral blood plasma samples by sandwich enzyme-linked immunosorbent assay (ELISA) in healthy controls $(n=12)$, in patients with $\operatorname{MGUS}(n=17)$, and in patients newly diagnosed with $(n=42)$ or relapsed/refractory $(n=25)$ MM. Results: Median endocan concentration increased from MGUS $(315.00 \mathrm{pg} / \mathrm{ml})$ and healthy controls $(316.19 \mathrm{pg} / \mathrm{ml})$ to newly-diagnosed $M M$ (371.82 pg/ml; $p=0.027)$. The low endocan levels (median=246.20 pg/ml) in patients with relapsed/refractory MM were similar to those in healthy controls and patients with MGUS. A cut-off value of $>220 \mathrm{pg} / \mathrm{ml}$ endocan in peripheral blood discriminated patients newly diagnosed with MM from those with MGUS (area under the curve $(A U C)=0.66,95 \%$ confidence interval $(C I)=0.55-0.81)$.
\end{abstract}

Correspondence to: Normann Steiner, MD, Ph.D., Department of Internal Medicine V, Innsbruck Medical University, Anichstr. 35, A-6020 Innsbruck, Austria. Tel: +43 51250482926, e-mail: normann.steiner@i-med.ac.at

Key Words: Multiple myeloma, endocan, biomarker.
Conclusion: The plasma levels of endocan can noninvasively differentiate patients newly diagnosed with MM from those with MGUS and should therefore be evaluated prospectively as a potential diagnostic marker.

Multiple myeloma (MM) evolves in a complex microenvironment of the bone marrow that supports growth, proliferation, migration and, ultimately, drug resistance of malignant plasma cells. In addition, interactions between myeloma cells and the bone marrow niche mediate bone destruction and angiogenesis, thereby contributing to MM pathogenesis and progression. Angiogenesis in bone marrow promotes nutrient and oxygen supply to myeloma cells (1, 2). Neovascularization in the marrow niche is a consistent hallmark of disease progression in plasma cell disorders and has a prognostic value (3). As shown in previous studies, increased bone marrow angiogenesis predicts poor survival in patients newly diagnosed with MM (4). Blood vessel formation is supported by various factors secreted by bone marrow stromal cells [e.g. vascular endothelial growth factor (VEGF) and interleukin 8 (IL8)] and MM cells [e.g. VEGF, basic fibroblast growth factor (bFGF) and matrix metalloproteinases (MMPs)]. Bone marrow endothelial cells secrete VEGF, IL6 and insulin-like growth factor 1 (IGF1), which stimulate MM cell growth $(5,6)$. Another cytokine, endocan, also referred to as endothelial cell-specific molecule-1 (ESM1), has been implicated in cell adhesion, tumor-associated inflammatory processes, angiogenesis, and tumor progression in various cancer types, such as renal, gastric, breast and lung $(7,8)$. Patients with late-stage lung cancer have high levels of endocan in their peripheral blood, 
Table I. Patient demographics and characteristics $(n=84)$.

\begin{tabular}{|c|c|c|c|}
\hline Parameter & $\begin{array}{l}\text { MGUS } \\
(\mathrm{n}=17)\end{array}$ & $\begin{array}{c}\mathrm{NDMM} \\
(\mathrm{n}=42)\end{array}$ & $\begin{array}{l}\text { RRMM } \\
(\mathrm{n}=25)\end{array}$ \\
\hline \multicolumn{4}{|l|}{ Age, years } \\
\hline Median (range) & $66(52-73)$ & $73(60-79)$ & $62(52-67)$ \\
\hline \multicolumn{4}{|l|}{ Gender, n (\%) } \\
\hline $\mathrm{F}$ & $5(29)$ & $19(45)$ & $12(48)$ \\
\hline M & $12(71)$ & $23(55)$ & $13(52)$ \\
\hline \multicolumn{4}{|l|}{ ISS, n (\%) } \\
\hline I & & $8(19)$ & $8(32)$ \\
\hline II & & $11(26)$ & $8(32)$ \\
\hline III & & $23(55)$ & $9(36)$ \\
\hline \multicolumn{4}{|c|}{$\begin{array}{l}\text { Type of Ig heavy chain } \\
\text { (serum), } \mathrm{n}(\%)\end{array}$} \\
\hline $\mathrm{IgG}$ & $14(82)$ & $21(50)$ & $17(68)$ \\
\hline $\operatorname{IgM}$ & $0(0)$ & $0(0)$ & $2(8)$ \\
\hline $\operatorname{IgA}$ & $2(12)$ & $8(19)$ & $1(4)$ \\
\hline $\mathrm{IgD}$ & $0(0)$ & $1(2)$ & $0(0)$ \\
\hline Light chain only & $1(6)$ & $12(29)$ & $5(20)$ \\
\hline \multicolumn{4}{|c|}{$\begin{array}{l}\text { Type of Ig light chain } \\
\text { (serum), } \mathrm{n}(\%)\end{array}$} \\
\hline Kappa & $10(59)$ & $27(64)$ & $15(60)$ \\
\hline Lambda & $7(41)$ & $15(36)$ & $10(40)$ \\
\hline \multicolumn{4}{|c|}{$\beta-2 \mathrm{M}$ icroglobulin, $\mathrm{n}(\%)$} \\
\hline$>\mathrm{UNV}$ & $11(69)$ & $33(83)$ & $19(76)$ \\
\hline \multicolumn{4}{|l|}{ LDH } \\
\hline$>\mathrm{UNV}$ & $3(43)$ & $6(14)$ & $8(32)$ \\
\hline \multicolumn{4}{|l|}{ Creatinine } \\
\hline$\geq 1.3 \mathrm{mg} / \mathrm{dl}$ & $7(41)$ & $25(60)$ & $10(40)$ \\
\hline \multicolumn{4}{|c|}{ Serum calcium, n (\%) } \\
\hline$>\mathrm{UNV}$ & $0(0)$ & $8(19)$ & $4(16)$ \\
\hline \multicolumn{4}{|l|}{ Hemoglobin, n (\%) } \\
\hline$\leq 12 \mathrm{~g} / \mathrm{dl}$ & $9(53)$ & $34(81)$ & $19(76)$ \\
\hline \multicolumn{4}{|l|}{ Platelets, n (\%) } \\
\hline$<100,000 / \mathrm{mm}^{3}$ & $2(12)$ & $10(24)$ & $15(60)$ \\
\hline \multicolumn{4}{|c|}{ Osteolytic bone lesions, n (\%) } \\
\hline Present & $1(6)$ & $35(83)$ & $25(100)$ \\
\hline \multicolumn{4}{|c|}{ Cytogenetic risk, n (\%) } \\
\hline Standard & $2(12)$ & $13(31)$ & $4(16)$ \\
\hline High & $2(12)$ & $20(48)$ & $17(68)$ \\
\hline Not available & $13(76)$ & $9(21)$ & $4(16)$ \\
\hline \multicolumn{4}{|c|}{ Plasma cells in BM (\%) } \\
\hline Median (range) & $3.2(0.7-6.5)$ & $30(7.3-70)$ & $50(18-80)$ \\
\hline \multicolumn{4}{|c|}{$\begin{array}{l}\text { Therapy lines at sample } \\
\text { collection, } \mathrm{n}(\%)\end{array}$} \\
\hline $1-2$ & $1(6)$ & $2(5)$ & $6(24)$ \\
\hline$\geq 4$ & $0(0)$ & $0(0)$ & $19(76)$ \\
\hline \multicolumn{4}{|l|}{ Therapy, n (\%) } \\
\hline PI-based & $0(0)$ & $0(0)$ & $11(44)$ \\
\hline IMiD-based & $1(6)$ & $2(5)$ & $8(32)$ \\
\hline PI + IMiD-based & $0(0)$ & $0(0)$ & $3(12)$ \\
\hline Other & $0(0)$ & $0(0)$ & $3(12)$ \\
\hline None & $16(4)$ & $40(95)$ & $0(0)$ \\
\hline
\end{tabular}

N, Number of patients; MGUS: monoclonal gammopathy of undetermined significance; NDMM newly diagnosed with multiple myeloma; RRMM: relapsed/refractory multiple myeloma; ISS, International Staging System (15); Ig; immunoglobulin; UNV upper normal value; $\mathrm{LDH}$, lactate dehydrogenase; IMiD, immunomodulatory drugs; PI, proteasome inhibitors; BM, bone marrow. which might reflect tumor angiogenic stimulation $(9,10)$. Endothelial cells produce and secrete endocan as a $50-\mathrm{kDa}$ soluble dermatan sulfate proteoglycan. Proangiogenic molecules such as VEGF and fibroblast growth factor (FGF2), as well as proinflammatory molecules, stimulate endocan secretion. The switch from dormant malignancies to exponentially growing tumors with an angiogenic phenotype was found to be closely associated with endocan (11). Previous studies proposed endocan as a novel prognostic biomarker based on its overexpression that correlated with tumor progression, metastasis and poor outcome $(10,12)$. Moreover, overexpression of endocan in human embryonic kidney cells induced tumor formation in SCID mice (13). Data on endocan expression and its potential role in patients with myeloma are still lacking.

Here, to our knowledge for the first time, plasma levels of endocan were analyzed in peripheral blood of patients with monoclonal gammopathy of undetermined significance (MGUS), those newly diagnosed with MM and those with relapsed/refractory MM.

\section{Materials and Methods}

Ethics statement. This research was conducted in accordance with the Declaration of Helsinki and was approved by the Authors' Institutional Review Board (number: AN2015-0034 346/4.13 Innsbruck, and 20/1/2011 Brno). Only patients who gave written informed consent were included.

Patients and sample collection. Healthy controls $(\mathrm{n}=12)$, patients with MGUS ( $n=17)$, those newly diagnosed with MM $(n=42)$ and patients with relapsed/refractory MM $(n=25)$ according to the International Myeloma Working Group criteria (14), were included. Patient characteristics are shown in Table I. Peripheral blood samples underwent centrifugation for $10 \mathrm{~min}$ at $1000 \times \mathrm{g}$ and the obtained plasma was collected and stored at $-80^{\circ} \mathrm{C}$.

Sandwich enzyme-linked immunosorbent assay (ELISA) for endocan. For the quantification of endocan concentration in plasma samples a commercially available solid-phase human endocan ELISA kit, purchased from ThermoFisher Scientific (Vienna, Austria), was used according to the manufacturer's instructions. Plasma samples, diluted 3-fold with the assay buffer, were incubated in duplicate in the ELISA plate pre-coated with specific capture antibody for $2.5 \mathrm{~h}$ at room temperature. Four washes were then performed to remove unbound proteins. Each well was then incubated with a biotin-labeled specific detection antibody at room temperature for 1 hour. After washing to remove unbound antibody, the plate was treated with a streptavidin-horseradish peroxidase conjugate for $45 \mathrm{~min}$. Following an enzymatic reaction with the substrate for peroxidase (room temperature, $30 \mathrm{~min}$ ) and subsequent termination of color development, absorbance at $450 \mathrm{~nm}$ and 550 $\mathrm{nm}$ was measured using an automated microplate reader.

Statistical analysis. Descriptive data are shown using median and interquartile range. Endocan levels between groups were compared using one-/two-factor analysis of variance (ANOVA). Welch test was 
used in the case of variance. Original $p$-values without corrections for multiple testing are provided. The Spearman correlation coefficient was used to test associations between quantitative variables. Sensitivity, specificity, predictive values and thresholds were calculated by receiver operating characteristic (ROC) analysis and the area under the ROC curves (AUCs) using Youden's approach. All tests for statistical significance were two-sided. Confidence intervals and $p$-values were calculated at a 5\% level of significance. Statistical evaluation was performed using SPSS statistical software (version 21.0; IBM Corp., Armonk, NY, USA) and OpenEpi Toolkit v3 (OpenEpi Project, Atlanta, GA, USA).

\section{Results}

The endocan level in peripheral blood is significantly elevated in patients newly diagnosed with MM. The endocan concentration in 96 plasma samples was determined. A significant difference in endocan expression was observed between healthy controls, MGUS, relapsed/refractory MM, and those newly diagnosed with MM ( $p<0.035$; Figure 1$)$. The lower endocan levels in peripheral blood in healthy controls and patients with MGUS (median of $316.19 \mathrm{pg} / \mathrm{ml}$ and $315.00 \mathrm{pg} / \mathrm{ml}$, respectively) were similar to those in patients newly diagnosed with MM, who had the highest endocan levels $($ median $=371.82 \mathrm{pg} / \mathrm{ml})$. Furthermore, those with relapsed/refractory MM had the lowest endocan levels (median=246.20 pg/ml). The ability of endocan to discriminate between these groups was determined by ROC analysis: patients newly diagnosed with $\mathrm{MM}$ versus all other groups gave an AUC of 0.68 [95\% confidence interval $(\mathrm{CI})=0.57-0.78]$.

Predictive value of endocan level for the identification of patients with newly-diagnosed MM. The ROC was calculated by plotting sensitivity against specificity for these groups and an optimal threshold was determined for discrimination between them. The plasma level of endocan discriminated MGUS patients from those newly diagnosed with MM (AUC $=0.66,95 \% \mathrm{CI}=0.55-0.81$ ). The score closest to the value under the peak sensitivity $(88 \%, 95 \% \mathrm{CI}=75-95 \%)$ and specificity $(39 \%, 95 \% \mathrm{CI}=20-61 \%)$ was calculated. A cut-off value of $>220 \mathrm{pg} / \mathrm{ml}$ for endocan was found to be suitable for identifying patients with undiagnosed MM. The positive predictive value (PPV) for a new diagnosis of MM was $77 \%$ (95\% CI=63-87\%) for patients with a level of endocan above $220 \mathrm{pg} / \mathrm{ml}$. The corresponding negative predictive value (NPV) was $58.3 \%(95 \% \mathrm{CI}=32-81 \%)$.

Significant association between endocan levels and clinical parameters and hypercalcemia, renal failure, anemia, and bone lesions (CRAB) criteria. Patient characteristics are shown in Table I. We found that patients with a higher disease stage, classified according to the International Staging System (14), had significantly higher endocan levels

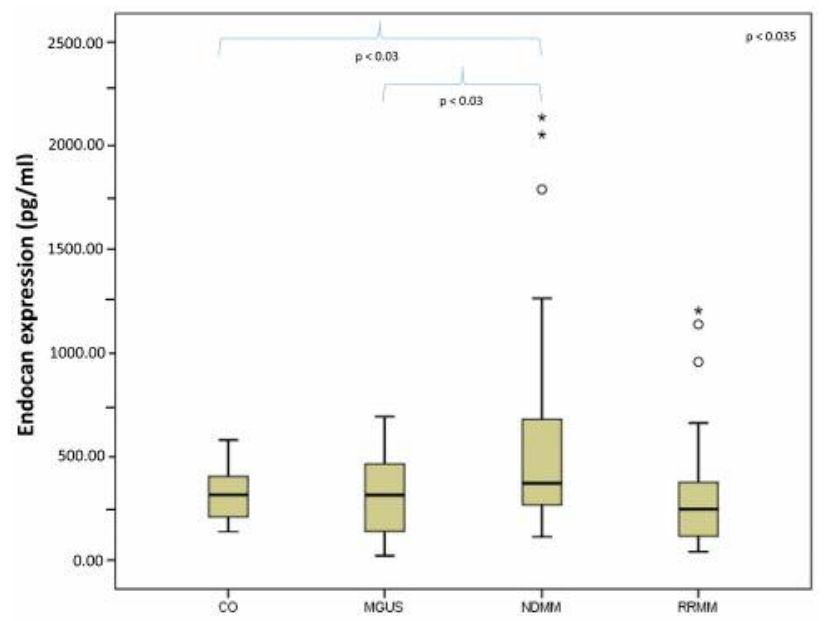

Figure 1. Endocan levels in peripheral blood of healthy controls $(\mathrm{CO})$, and patients with monoclonal gammopathy of unknown significance (MGUS), relapsed refractory multiple myeloma (RRMM), and newlydiagnosed multiple myeloma (NDMM). The low endocan levels seen in peripheral blood of healthy controls (CO) and patients with MGUS were similar, in contrast to those with NDMM, who had the highest endocan levels. Patients with RRMM had the lowest endocan levels. $p$ Values below a significance level of $\alpha=0.05$ were considered statistically significant. The dark line in the middle of the boxes is the median. Half of the cases/rows have a value greater than the median, and half have a value lower. The bottom of the box indicates the 25th percentile. Twenty-five percent of cases/rows have values below the 25th percentile. The top of the box represents the 75th percentile. Twenty-five percent of cases/rows have values above the 75th percentile. This means that 50\% of the case/rows lie within the box. The T-bars that extend from the boxes extend to 1.5 times the height of the box or, if no caselrow has a value in that range, to the minimum or maximum values. The dots/asterisks represent cases that have values more than three times the height of the boxes.

$(p<0.001$, Figure 2A). Patients with immunoglobulin lambda light chains also had elevated endocan levels in comparison to patients with kappa light chains $(p<0.003$, Figure $2 \mathrm{~B})$. Moreover, high $\beta 2$ microglobulin and lactate dehydrogenase levels were also associated with high endocan expression ( $p<0.001$, Figure 2C and $p<0.009$, Figure 2D, respectively), and elevated creatinine and anemia were strongly linked to higher endocan secretion in patients with MM $(p<0.001$, Figure $2 \mathrm{E}$ and $p<0.009$, Figure $2 \mathrm{~F}$ ). No significant association was observed in patients with elevated serum $\mathrm{Ca}^{2+}$ level and osteolytic bone lesions. A significant weak correlation was found between endocan level and the percentage of plasma cells in the bone marrow $(\mathrm{rh}=0.3$, $p<0.04)$. Patients treated with immunomodulatory agentbased therapies had stronger endocan expression compared to than did patients receiving proteasome inhibitor-based therapies, but the difference was not significant $(p<0.2)$. No associations were found regarding therapy lines, cytogenetics, age/sex, and type of immunoglobulin status. 

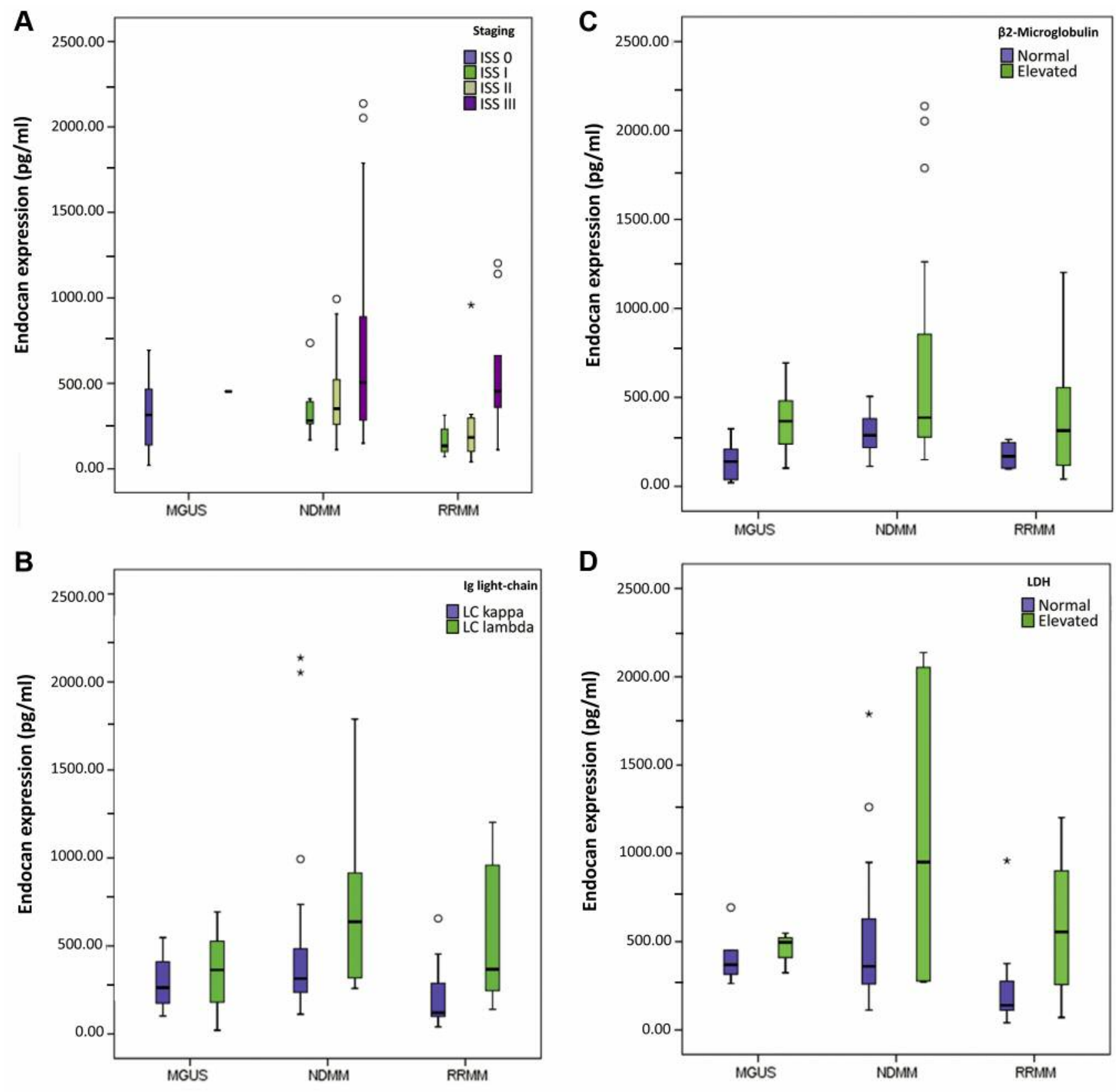

Figure 2. Continued

\section{Discussion}

The prognosis of patients with MM has substantially improved due to implementation of novel agents such as new proteasome inhibitors, immunomodulatory drugs and monoclonal antibodies. Nevertheless, no predictive markers are currently available for the detection of early progression from MGUS to symptomatic MM.

The aim of this study was to evaluate whether the circulating dermatan sulfate proteoglycan endocan can be

used to detect early progression of MGUS to MM. The strength of our work lies in the large sample number encompassing various cohorts and healthy controls. A potential limitation is the unavailability of follow-up samples.

Endocan plays an important role in cell adhesion and tumorassociated inflammation. Moreover, it is correlated with poor progression-free survival in various cancer types and can reflect tumor angiogenic stimulation $(7,8,15,16)$. We found high levels of endocan in peripheral blood of patients with 

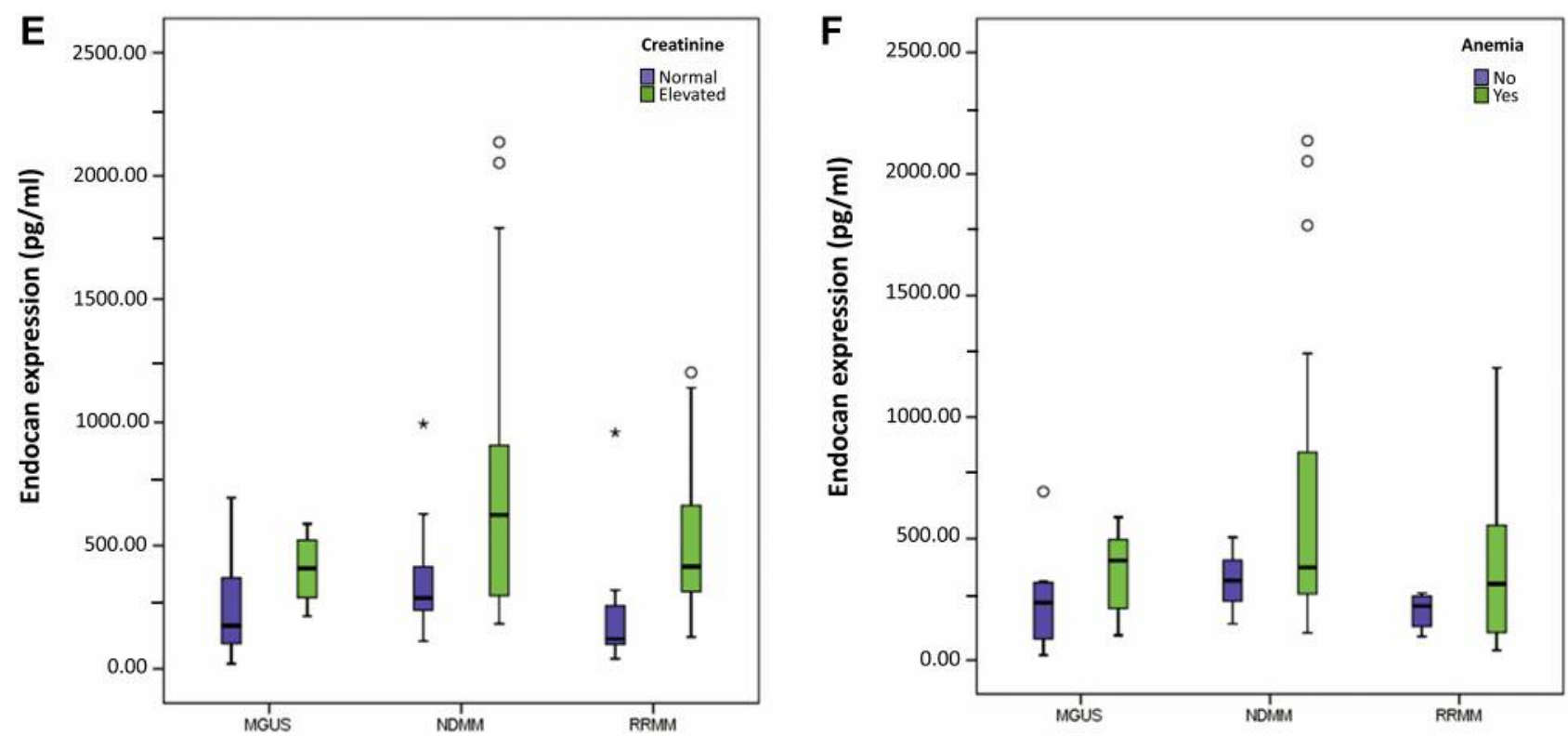

Figure 2. Associations between endocan levels in peripheral blood of healthy controls $(\mathrm{CO})$, and patients with monoclonal gammopathy of unknown significance (MGUS), relapsed refractory multiple myeloma (RRMM), and newly-diagnosed multiple myeloma (NDMM) according to stage (A), immunoglobulin light chain $(L C)(B)$, and levels of $\beta 2$ microglobulin $(C)$, lactate dehydrogenase $(L D H)(D)$, creatinine $(E)$, and anemia $(F)[c l i n i c a l$ parameters of CRAB criteria (hypercalcemia, renal failure, anemia, and bone lesions)]. A: Patients with NDMM and those with RRMM with a higher disease stage had significantly higher endocan levels. B: Patients with immunoglobulin lambda light chains had elevated endocan levels as compared to those with kappa light chains. C: Patients with high $\beta 2$ microglobulin levels had elevated endocan levels. D: Patients with high lactate dehydrogenase levels had elevated endocan levels. E: Patients with high creatinine values had elevated endocan levels. F: Patients with anemia had elevated endocan levels. The dark line in the middle of the boxes is the median. Half of the cases/rows have a value greater than the median, and half have a value lower. The bottom of the box indicates the 25th percentile. Twenty-five percent of cases/rows have values below the 25 th percentile. The top of the box represents the 75th percentile. Twenty-five percent of cases/rows have values above the 75th percentile. This means that 50\% of the case/rows lie within the box. The T-bars that extend from the boxes extend to 1.5 times the height of the box or, if no case/row has a value in that range, to the minimum or maximum values. The dots/asterisks represent cases that have values more than three times the height of the boxes.

newly diagnosed MM, where a threshold of $>220 \mathrm{pg} / \mathrm{ml}$ identified those patients. The PPV was $77 \%$ for newlydiagnosed MM. This finding suggests endocan as a marker for identification of progression of MGUS to MM. A high endocan concentration at progression from MGUS to symptomatic MM might be caused by greater endothelial and perivascular cell infiltration in patients newly diagnosed with MM. Bone marrow-infiltrating cells secrete angiogenic molecules, cytokines and chemokines, which may potentially stimulate endocan overexpression. An additional increase in endocan in those newly diagnosed with MM may be caused by secretion of VEGF by malignant plasma cells and their strong interaction with the bone marrow microenvironment (17-19).

However, as observed in our cohort, endocan was not suitable for detection of a relapse or refractory MM. A potential explanation for this finding could be reduced angiogenic activity in the bone marrow of these relapsed/heavily pretreated patients with MM, in particular after proteasome inhibitor-based therapies. A decrease in angiogenesis in relapsed/refractory $\mathrm{MM}$ results in less secretion of angiogenic molecules and cytokines in peripheral blood (5), which presumably results in a lower level of circulating endocan.

Moreover, our analyses of endocan levels and myelomaspecific clinical parameters showed that a high level of endocan was significantly associated with elevated lactate dehydrogenase, $\beta 2$ microglobulin and creatinine levels, and anemia. These results suggest the potential implementation of endocan into diagnostic criteria to improve the early detection of symptomatic MM. We found that patients subjected to immunomodulatory agent-based regimen had a higher endocan level than did those treated with proteasome inhibitors. A potential reason for this could be the involvement of immunomodulatory agents in the antiangiogenic process in MM, resulting in higher endocan expression in peripheral blood. Due to the small number of participants in both therapy cohorts, these differences were not statistically significant.

Next, the significant weak correlation between endocan level and the number of plasma cells in the bone marrow can 
be explained by the elevated plasma cell counts in patients with MM as compared to those with MGUS.

In conclusion, as far as we are aware our study is the first to show the diagnostic value of endocan in plasma samples of peripheral blood in four different cohorts (MGUS, newly diagnosed with MM, relapsed/refractory MM, and healthy participants). We found that a high level of circulating endocan was significantly associated with myeloma-specific clinical parameters. Moreover, an increased endocan level and values above a threshold of $220 \mathrm{pg} / \mathrm{ml}$ correlate with progression from MGUS to symptomatic MM. Furthermore, this marker should also be examined with regard to treatment response in patients with MM. Based on the novelty of this proteoglycan in MM and its significantly higher expression in patients newly diagnosed with MM than in those with MGUS, our data suggest that endocan might be useful for identifying patients with progression to MM. Therefore, our findings may serve as a basis for further detailed evaluation of endocan as a potential diagnostic marker.

\section{Acknowledgements}

The Authors thank Cornelia Heis for her excellent technical assistance.

\section{References}

1 Rajkumar SV, Mesa RA, Fonseca R, Schroeder G, Plevak MF, Dispenzieri A, Lacy MQ, Lust JA, Witzig TE, Gertz MA, Kyle RA, Russell SJ and Greipp PR: Bone marrow angiogenesis in 400 patients with monoclonal gammopathy of undetermined significance, multiple myeloma, and primary amyloidosis. Clin Cancer Res 8: 2210-226, 2002.

2 Palumbo A and Anderson K: Multiple myeloma. N Engl J Med 364: 1046-1060, 2011

3 Ntellas P, Perivoliotis K, Dadouli K, Koukoulis GK and Ioannou $\mathrm{M}$ : Microvessel density as a surrogate prognostic marker in patients with multiple myeloma: A meta-analysis. Acta Haematologica 138: 77-84, 2017.

4 Rajkumar SV, Leong T, Roche PC, Fonseca R, Dispenzieri A, Lacy MQ, Lust JA, Witzig TE, Kyle RA, Gertz MA and Greipp PR: Prognostic value of bone marrow angiogenesis in multiple myeloma.Clin Cancer Res 6: 3111-3116, 2000.

5 Roccaro AM, Hideshima T, Raje N, Kumar S, Ishitsuka K, Yasui H, Shiraishi N, Ribatti D, Nico B, Vacca A, Dammacco F, Richardson PG and Anderson KC: Bortezomib mediates antiangiogenesis in multiple myeloma via direct and indirect effects on endothelial cells. Cancer Res 66: 184-191, 2006.

6 Gupta D, Treon SP, Shima Y, Hideshima T, Podar K, Tai YT, Lin B, Lentzsch S, Davies FE, Chauhan D, Schlossman RL, Richardson P, Ralph P, Wu L, Payvandi F, Muller G, Stirling DI and Anderson KC: Adherence of multiple myeloma cells to bone marrow stromal cells up-regulates vascular endothelial growth factor secretion: therapeutic applications. Leukemia 15: 19501961, 2001.
7 Leroy X, Aubert S, Zini L, Franquet H, Kervoaze G, Villers A, Delehedde M, Copin MC and Lassalle P: Vascular endocan (ESM-1) is markedly overexpressed in clear cell renal cell carcinoma. Histopathology 56: 180-187, 2010.

8 Zhao W, Sun M, Li S, Wang Y and Liu J: Biological and clinical implications of endocan in gastric cancer. Tumour Biol 35: 10043-9, 2014

9 Huang GW, Tao YM and Ding X: Endocan expression correlated with poor survival in human hepatocellular carcinoma. Dig Dis Sci 54: 389-394, 2009.

10 Grigoriu BD, Depontieu F, Scherpereel A, Gourcerol D, Devos P, Ouatas T, Lafitte JJ, Copin MC, Tonnel AB and Lassalle P: Endocan expression and relationship with survival in human non-small cell lung cancer. Clin Cancer Res 12: 4575-4582, 2006.

11 Almog N, Ma L, Raychowdhury R, Schwager C, Erber R, Short S, Hlatky L, Vajkoczy P, Huber PE, Folkman J and Abdollahi A: Transcriptional switch of dormant tumors to fast-growing angiogenic phenotype. Cancer Res 69: 836-844, 2009.

12 Sagara A, Igarashi K, Otsuka M, Kodama A, Yamashita M, Sugiura R, Karasawa T, Arakawa K, Narita M, Kuzumaki N, Narita $\mathrm{M}$ and Kato Y: Endocan as a prognostic biomarker of triple-negative breast cancer. Breast Cancer Res Treatm 161: 269-278, 2017.

13 Scherpereel A, Gentina T, Grigoriu B, Senechal S, Janin A, Tsicopoulos A, Plenat F, Bechard D, Tonnel AB and Lassalle P: Overexpression of endocan induces tumor formation. Cancer Res 63: 6084-6089, 2003.

14 Group IMW: Criteria for the classification of monoclonal gammopathies, multiple myeloma and related disorders: a report of the International Myeloma Working Group. Br J Haematol 121: 749-757, 2003.

15 Cornelius A, Cortet-Rudelli C, Assaker R, Kerdraon O, Gevaert MH, Prevot V, Lassalle P, Trouillas J, Delehedde M and Maurage CA: Endothelial expression of endocan is strongly associated with tumor progression in pituitary adenoma. Brain Pathol 22: 757-764, 2012.

16 Lin LY, Yeh YC, Chu CH, Won JGS, Shyr YM, Chao Y, Li CP, Wang SE and Chen MH: Endocan expression is correlated with poor progression-free survival in patients with pancreatic neuroendocrine tumors. Medicine 96: e8262, 2017.

17 Ribatti D, Nico B and Vacca A: Importance of the bone marrow microenvironment in inducing the angiogenic response in multiple myeloma. Oncogene 25: 4257-4266, 2006.

18 Ribatti D, Mangialardi G and Vacca A: Antiangiogenic therapeutic approaches in multiple myeloma. Curr Cancer Drug Targ 12: 768-775, 2012.

19 Ferrarini $\mathrm{M}$ and Ferrero E: Proteasome inhibitors and modulators of angiogenesis in multiple myeloma. Curr Med Chem 18: 51855195, 2011. 